\title{
The molten globule state of $\alpha$-lactalbumin
}

\author{
KUNIHIRO KUWAJIMA ${ }^{1}$ \\ Department of Physics, School of Science, University of Tokyo, 7-3-1 Hongo, Bunkyo-ku, Tokyo 113, Japan
}

ABSTRACT The molten globule state of $\alpha$-lactalbumin is the best-characterized folding intermediate of globular proteins and has been studied intensively by various spectroscopic and physicochemical techniques, including stopped-flow $\mathrm{CD}$ and fluorescence spectroscopies, a hydrogen-exchange technique, ${ }^{1} \mathrm{H}$ NMR spectroscopy, disulfide-exchange chemistry, site-directed mutagenesis, and calorimetric techniques. This review summarizes recent studies. Major findings about the structure of the molten globule state are: 1) It is highly heterogeneous, having a highly structured $\alpha$-helical domain with the $\beta$-sheet domain being significantly unfolded; and 2 ) it is not a nonspecific, collapsed polypeptide but already has a native-like tertiary fold. These structural characteristics are essential to fully understand the thermodynamic properties of the molten globule state, which are described in connection with a recently proposed computational approach to predict the structure of the molten globule state of a protein. Mutant proteins in which the stability of the molten globule state was changed were constructed. Studies of the equilibrium unfolding and kinetic refolding of the mutant proteins will provide further insight into the molten globule state as a folding intermediate. In spite of an initial expectation that the structure recognized by an Escherichia coli chaperone, GroEL, is the molten globule, the interaction of GroEL with $\alpha$-lactalbumin in the molten globule state is much weaker than the interaction with more unfolded states of $\alpha$-lactalbumin, a disulfide-reduced form, and disulfide rearranged species.-Kuwajima, K. The molten globule state of $\alpha$-lactalbumin.-FASEB J. 10, 102-109 (1996)

Key Words: folding intermediate $\cdot$ protein folding $\cdot$ molecular chaperone

THE MOST ESSENTIAL QUESTION IN protein folding studies is how an unstructured polypeptide of a protein can fold into its unique native conformation in a reasonable period of time even though there are an astronomically large number of possible conformational states (1). This socalled Levinthal's paradox has directed researchers in this field to try to detect and characterize possible intermediates between the native and fully unfolded states (2-11). The presence of a unique pathway of folding, on which a limited number of specific intermediates are well populated, seems to provide an answer to the paradox.
Our understanding of the folding intermediates has made great advances during the last 20 years. Intermediates that were characterized in only a few proteins 20 years ago (12) were regarded as rather exceptional $(13,14)$. Now we find many reports on the structure of the folding intermediates even at a level of atomic resolution. The availability of new experimental techniques was responsible for this advance in protein folding studies. Studies by stopped-flow circular dichroism (CD) ${ }^{2}$ techniques have unequivocally shown the presence of kinetic refolding intermediates, which have appreciable amounts of secondary structure and accumulate within $10 \mathrm{~ms}$ after the refolding starts in a globular protein $(7,15,16)$. Hydrogen-exchange labeling combined with 2D NMR spectroscopy has provided a powerful technique by which we can characterize the structure of refolding intermediates at the level of amino acid residue resolution $(6,17)$.

Many excellent reviews have appeared on folding intermediates of globular proteins characterized by the advanced techniques mentioned above $(6,7,15-21)$. This review therefore does not attempt to be so comprehensive as to include all aspects of kinetic folding intermediates. It confines the subject to the molten globule state of $\alpha$ lactalbumin because this intermediate has been studied intensively in the last few years. $\alpha$-Lactalbumin displays the best-characterized molten globule state and is the best model protein in protein folding studies.

\section{$\alpha$-LACTALBUMIN}

$\alpha$-Lactalbumin is a small $\mathrm{Ca}^{2+}$-binding protein $(14,200$ Da) present in mammalian milk and functions as a specificity modifier of an enzyme, galactosyltransferase (22-24). It is genetically and structurally homologous to c-type lysozyme $(22,24-26)$, and its high-resolution Xray crystallographic structure has already been reported (27-30) (Fig. 1). The structure of $\alpha$-lactalbumin consists of two subdomains: an $\alpha$-helical domain and a $\beta$-sheet domain. There are four $\alpha$-helices-A [residues 5-11], B [23-34], C [86-99], and D [105-109]-and three 310helices [12-16, 101-104, and 115-119] in the $\alpha$-helical

\footnotetext{
${ }^{1}$ Address for correspondence and reprint requests to Dr. Kuwajima, at: Department of Physics, School of Science, University of Tokyo, 7-3-1 Hongo, Bunkyo-ku, Tokyo 113, Japan.

${ }^{2}$ Abbreviations: CD, circular dichroism; 2D NMR, 2-dimensional NMR; GdnHCl, guanidine hydrochloride; 2D NOESY, 2-dimensional nuclear Overhauser effect spectroscopy.
} 
domain; and an anti-parallel $\beta$-sheet $[40-50]$ and a $310-$ helix $[76-82]$ in the $\beta$-sheet domain. The structural and functional properties of $\alpha$-lactalbumin and its interrelationships with lysozyme are excellently reviewed by McKenzie and White (24) and by Kronman (31). A remarkable property of $\alpha$-lactalbumin as a model of protein folding studies is the high stability of its molten globule state (4), which is observed in the following conditions (4): 1) an equilibrium unfolding intermediate at a moderate concentration of a strong denaturant [guanidine hydrochloride $(\mathrm{GdnHCl})$ or urea], 2) the acid-denatured state, and 3) a partially unfolded state produced by removal of the bound $\mathrm{Ca}^{2+}$ at neutral $\mathrm{pH}$ and low salt concentration. The known structural characteristics of the molten globule state are: 1) native-like secondary structure, 2) compact structure with a radius only 10-20\% larger than that of the native molecule, and 3) the absence of the specific tertiary packing interactions of amino acid side chains (4). Our group studied the equilibrium and kinetics of the unfolding-refolding reactions among the three states-the native, the molten globule, and the fully unfolded states $(12,32,33)$-and proposed a folding model based on the three-state unfolding of $\alpha$ lactalbumin (34). This model was later verified, using a kinetic CD technique $(35,36)$, by direct observation of a transient refolding intermediate that is identical with the molten globule state. These studies have been reviewed by the author $(4,15,16,37)$ and by Sugai and Ikeguchi (38). In this review, only the most recent progress in the studies of the molten globule state of $\alpha$-lactalbumin will be described.

\section{HOW NATIVE-LIKE IS THE MOLTEN GLOBULE?}

Although the molten globule state has native-like secondary structure as measured by the far UV CD spectra, it is important to ask whether the locations of the secondary structure segments along the polypeptide chain are the same as in the native state. Dobson and co-workers (39-41) used ${ }^{1} \mathrm{H}-\mathrm{NMR}$ spectroscopy and a hydrogen exchange technique for investigating the structure of the molten globule state of $\alpha$-lactalbumin, finding that the B and $C$ helices are formed and have protected amide protons. The structure of the molten globule is highly heterogeneous, having the highly structured $\alpha$-helical domain formed by loose hydrophobic interactions whereas the $\beta$ sheet domain is significantly more unfolded. This picture of the molten globule state, organization of the native secondary structure segments in a part of a protein molecule by loose tertiary contacts, is also revealed in other globular proteins by the hydrogen-exchange technique combined with ${ }^{1} \mathrm{H}$-NMR spectroscopy $(6,17,19,42-46)$. It is, however, important to note that the hydrogen exchange technique can detect only native secondary structure. The amide protons protected in the nonnative secondary structure, if present, will be exchanged out during the

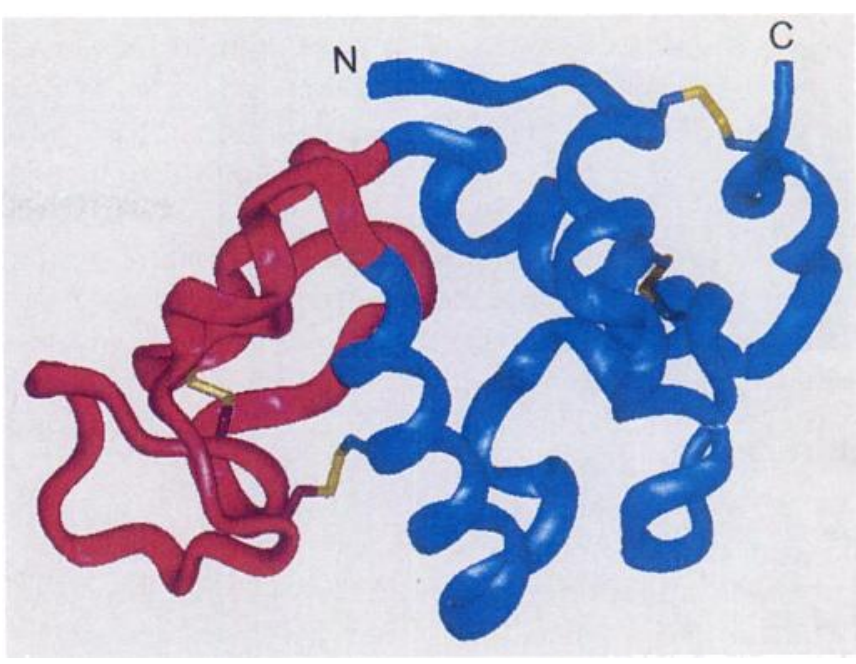

Figure 1. Schematic representation of the structure of baboon $\alpha$-lactalbumin generated from the structure proposed by Acharya et al. (27), using coordinates 1ALC of the Brookhaven Protein Data Bank. The $\alpha$-helical domain (residues 1-37 and 85-123) is shown in blue and the $\delta$-sheet domain (residues 38-84) in red. Four yellow sticks indicate the four disulfide bonds of the protein.

NMR measurement done in the native state. Formation of nonnative $\alpha$-helices at an early stage of refolding has heen suggested in $\beta$-lactoglobulin by equilibrium and kinetic CD studies $(16,47)$.

Alexandrescu et al. have reported the 2-dimensional nuclear Overhauser effect spectroscopy (2D NOESY) spectra of $\alpha$-lactalbumin in the molten globule state (40). They have shown that the side chains of Tyr ${ }^{103}, \operatorname{Trp}^{104}$, and $\mathrm{His}^{107}$ form a hydrophobic cluster in the molten globule state, but that the structure in this region may differ from the corresponding region in the fully native protein. Hydrogen exchange of tryptophan indole protons, photochemically induced dynamic nuclear polarization, and paramagnetic perturbation of the NMR spectra have also shown that $\operatorname{Trp}^{26}$ and $\operatorname{Trp}^{104}$, buried in the native state, are buried in the molten globule $(41,48)$ so that some form of a hydrophobic core in the $\alpha$-helical domain of the native structure may persist in the molten globule state. Hamada et al. (49) have reported that the cleavage of the peptide bond between Asp ${ }^{66}$ and $\mathrm{Pro}^{67}$ located at the edge of the $\beta$-sheet domain makes the $\alpha$-lactalbumin molecule characteristic of the molten globule with respect to CD and fluorescence spectra and 1-anilino-naphthalene-8sulfonate (ANS) binding. Nölting et al. (50) have shown the presence of significant conformational relaxation at a time scale faster than $\mathbf{5 0 0} \mathbf{~ n s}$ in the acidic molten globule state by ultrasonic velocimetry.

Another important issue as to the structure of the molten globule state is: Is it a nonspecific collapsed polypeptide or does it already have a native-like tertiary fold? Ikeguchi et al. (51) found that selective reduction of the 6-120 disulfide bond of bovine $\alpha$-lactalbumin reduces the stability of the molten globule state by $0.8 \sim 1.2$ $\mathrm{kcal} / \mathrm{mol}$, and have suggested that some ordered structure is present within the loop moiety formed by this disulfide. 
Creighton and Ewbank (52-54) have studied the threedisulfide species of human $\alpha$-lactalbumin and found that the three-disulfide form with free thiols on $\mathrm{Cys}^{6}$ and Cys ${ }^{120}$ can adopt the molten globule conformation and then spontaneously rearrange its three disulfide bonds to many isomers that maintain similar conformations with respect to spectroscopic and hydrodynamic properties. They concluded that the molten globule state of $\alpha$-lactalbumin does not maintain the native-like topology of the polypeptide backbone but is more like a collapsed form of an unfolded protein.

A series of recent papers by $\mathrm{Kim}$ and co-workers (55-58) also illuminates this issue, although their conclusion is apparently contradictory to that drawn by Creighton and Ewbank (54). They constructed a singlechain, recombinant model of the $\alpha$-helical domain of human $\alpha$-lactalbumin, the $\alpha$-domain, which consists of residues 1-39 and 81-123 of $\alpha$-lactalbumin connected by a short linker of three glycines (55). The $\alpha$-domain that has two native disulfide bonds (6-120 and 28-111) shows characteristics of the molten globule state with respect to the $C D$ and ${ }^{~} \mathrm{H}-\mathrm{NMR}$ spectra and the diffuse thermal transition, showing that this mini-"protein" cannot form a rigid tertiary structure under the same condition that the intact protein forms the native structure. The disulfideexchange reaction of $\alpha$-domain in a redox buffer solution, however, produced predominantly the native disulfide bonds, although the other two nonnative disulfides have much higher probabilities if they are formed in a randompairing model of a fully unfolded polypeptide. The results clearly indicate that $\alpha$-domain has a native-like tertiary fold in spite of its molten globule characteristics. They have also studied two kinds of the full-length species [ $\alpha$ $L A(\alpha)$ and $\alpha-L A(\beta)]$ (56). $\alpha-L A(\alpha)$ contains the same two disulfide bonds as in the $\alpha$-domain, with the cysteines for the other two disulfides replaced by alanines, whereas $\alpha-\operatorname{LA}(\beta)$ contains the 61-77 disulfide in the $\beta$ sheet domain and the interdomain 73-91 disulfide bond, with the cysteines in the $\alpha$-helical domain replaced by alanines. $\alpha-L A(\alpha)$ shows the same equilibrium preferences of formation of the native disulfides as observed in the $\alpha$-domain, but $\alpha-L A(\beta)$ does not show a strong preference for the native disulfide formation. Thus, the molten globule state of $\alpha$-lactalbumin has a heterogeneous structure in which the $\alpha$-helical domain resembles an expanded native-like protein and the $\beta$-sheet domain is largely unstructured. These results are consistent with the hydrogen exchange results of Dobson and co-workers (41) and also with the study of the three-disulfide species by Ikeguchi et al. (51). The apparent contradiction with the results of Creighton and Ewbank (54) may be interpreted in terms of differences in the number of possible disulfide pairings between the proteins used by the two groups. Intact $\alpha$-lactalbumin, used by Creighton's group, contains all eight cysteines, so that the preference for the native disulfides in the $\alpha$-helical domain may be obscured by much more random distribution of disulfide pairings possible in the intact protein.
Peng et al. $(57,58)$ have recently reported the structural specificity of local regions surrounding specific disulfide bonds in the molten globule state. They determined the effective concentrations for disulfide bond formation for the two native disulfide bonds (6-120 and 28-111) in the $\alpha$-helical domain and four nonnative disulfides $(6-28,6-111,28-120$, and 111-120) by using the single-disulfide mutants of full-length $\alpha$-lactalbumin. The effective concentration for formation of the native 28-111 disulfide bond was more than 10-fold higher than the concentration for formation of any other native or nonnative disulfide bond and more than 1000 -fold higher than the concentration expected in the random pairing model. Thus, the local region surrounding the 28-111 disulfide bond has a high preference for adopting a native-like structure in the molten globule state.

\section{THERMODYNAMIC PROPERTIES}

A remarkable property of the molten globule state of $\alpha$ lactalbumin is its diffuse thermal transition. Apparently, there is no cooperative heat absorption peak in scanning calorimetric measurements when the heating is started from the molten globule state at acid $\mathrm{pH}(35,59,60)$. This observation suggests that the molten globule and the thermally unfolded states are thermodynamically indistinguishable, so that there is no thermal unfolding when we start from the molten globule state. In fact, the thermally unfolded state of $\alpha$-lactalbumin shares common characteristics with the molten globule state-i.e., the nativelike secondary structure and the compactness of the molecule-especially when the thermal unfolding is realized at a relatively low temperature by destabilizing the native state $(35,60,61)$.

Physical interpretation of the absence of the cooperative thermal transition of the molten globule state has, however, been controversial. By using multidimensional analysis of the heat capacity surface obtained from scanning calorimetric measurements of $\alpha$-lactalbumin at different concentrations of $\mathrm{GdnHCl}$, Xie et al. (62) have suggested that the intrinsic enthalpy change from the molten globule to the thermally unfolded state must be much higher $\left(24 \mathrm{kcal} / \mathrm{mol}\right.$ at $\left.25^{\circ} \mathrm{C}\right)$ than that observed experimentally in the presence of $\mathrm{GdnHCl}$ or at an acid $\mathrm{pH}$; it is even higher than the enthalpy change from the native to the molten globule state (only $8 \mathrm{kcal} / \mathrm{mol}$ by their estimate at $25^{\circ} \mathrm{C}$ ). This conclusion has, however, been disputed by Yutani et al. (63), who have examined the heat capacity function of apo- $\alpha$-lactalbumin in the molten globule state at neutral $\mathrm{pH}$ without $\mathrm{GdnHCl}$ by differential scanning microcalorimetry and have shown the absence of the thermal transition. Although Xie et al. (64), opposing the conclusion of Yutani et al. (63), later attributed the absence of the thermal transition to ionic strength dependence of the thermal transition temperature, their argument is misleading because the thermal transition observed at a high ionic strength is not the 
transition from the molten globule state but rather the transition from the native to the molten globule state (65).

An extensive study of the energetics of the $\alpha$-lactalbumin states has recently been reported by Griko et al. (66). At $\mathrm{pH}$ values close to neutral, the thermal unfolding from the native state occurs at high temperature and yields a fully unfolded polypeptide with no measurable population of partly folded intermediates. At lower $\mathrm{pH}$ values, however, the unfolding occurs at lower temperatures, and a progressively higher population of the molten globule-like intermediates was observed. Because apo- $\alpha$ lactalbumin unfolds at a low temperature even at a neutral $\mathrm{pH}$, their observation of the population of the molten globule after the thermal unfolding is consistent with the results of Yutani et al. (63). Griko et al. (66) have suggested that the molten globule state has a higher enthalpy than the unfolded state below $45^{\circ} \mathrm{C}$, so that when the thermal unfolding occurs below $45^{\circ} \mathrm{C}$ the thermally unfolded state corresponds to the molten globule state, and once the molten globule is formed, the structure of the protein gradually unfolds upon heating and shows a gradual increase in heat capacity.

The unfolding of the molten globule state induced by a denaturant, urea or $\mathrm{GdnHCl}$, is, however, known to show a cooperative transition, and Ptitsyn and Uversky (67) recently demonstrated that the transition from the molten globule to the fully unfolded state is a two-state transition. The thermal unfolding of $\alpha$-lactalbumin has also been studied by fluorescence spectroscopy and binding of a hydrophobic fluorophor (68-72). Shimizu et al. (73) have studied the urea-induced unfolding of the molten globule state of $\alpha$-lactalbumin by titration of aromatic resonances in the ${ }^{1} \mathrm{H}-\mathrm{NMR}$ spectra and demonstrated that the unfolding of the molten globule is not a cooperative two-state process.

Xie and Freire (74) have recently investigated partly folded structures of $\alpha$-lactalbumin, which are consistent with the experimental thermodynamic data, by an interesting computational approach. They generated many partly folded structures from the known X-ray crystallographic structure of $\alpha$-lactalbumin, using a combinatorial unfolding algorithm. The thermodynamic properties of the partly folded states have been predicted by calculating the accessible surface area and by using an empirically derived structural parameterization. Partly folded states were chosen that satisfy the thermodynamic properties of the molten globule state. The criteria used to choose these states are a lower heat capacity and a higher enthalpy at low temperatures than those in the fully unfolded state $(66,74)$. Several partly folded states were chosen. All of them have the A, B, and C helices folded, whereas the $\beta$-sheet and the $D$ helix were unfolded. Their results are thus consistent with the NMR and hydrogen exchange studies by Dobson's group (41), who have shown the $B$ and $C$ helices to be formed in the molten globule state. Their results are, however, not fully consistent with the results of Peng et al. (58), who have shown that the region surrounding the 28-111 disulfide bond, which may include the $\mathrm{D}$ helix, is formed in the molten globule state. In all their calculations, Xie and Freire (74) have assumed that the folded regions in the partly folded states preserve the conformation that exists in the native structure. This is clearly an oversimplification. Quantitative agreement in the thermodynamic parameters between the theory and experiment, they have reported, is thus rather surprising. They have recently applied their computational approach to investigating the molten globule states of other globular proteins (75-77).

Studies of the $\alpha$-lactalbumin mutants in which the stability of the molten globule state is changed by the mutations will be very useful for elucidating the molecular mechanism of stabilization of the molten globule state. Recently, Uchiyama et al. (78) have reported studies of such mutants of goat $\alpha$-lactalbumin. They introduced a Thr to Ile mutation at residues 29 and 33, and a Ala to Ile or Ala to $\mathrm{Thr}$ mutation at residue 30 . The mutation sites are located in the $B$ helix, and the mutations change the hydrophobicity of the core of the $\alpha$-helical domain formed by the $B$ helix. The choice of these mutation sites is based on the fact that guinea pig $\alpha$-lactalbumin shows the most stable molten globule state among the three $\alpha$ lactalbumin species from goat, bovine, and guinea pig. The stabilization free energies of the molten globule state have been estimated to be $3.1,1.5$, and $0.7 \mathrm{kcal} / \mathrm{mol}$ for the guinea pig, bovine, and goat proteins, respectively (78). The amino acid residues at these sites are all threonines in the bovine protein; residue 30 is replaced by an alanine in the goat protein, with the other two residues being the same as in the bovine protein, and the three residues are all replaced by hydrophobic isoleucines in the guinea pig protein. The stability of the molten globule state of the five mutants (T29I, A30T, A30I, T33I, and A30I+T33I) of goat $\alpha$-lactalbumin has been investigated by urea-induced unfolding transition at pH 2.0, where the molten globule state is stable without urea. The molten globule state is stabilized by the amino acid substitutions that raise the hydrophobicity of the residues, suggesting that the hydrophobic core in a globular protein plays an important role in stabilization of the molten globule state. The increase in stability of the molten globule state is much smaller than the difference in stability between the guinea pig and goat proteins and less than $10 \%$ of the change expected from the transfer free energy of amino acids from water to hydrophobic environment, e.g., the A30I+T33I double mutation increases the stability of the molten globule state only by $0.5 \mathrm{kcal} / \mathrm{mol}$ (78). Thus, the amino acid substitutions other than those at the three sites just referred to are also responsible for the remarkable stability of the molten globule state of the guinea pig protein. It has also been concluded that the hydrophobic core in the molten globule state is highly hydrated and much looser than the core of the native molecule (78).

Pardon et al. (79) have recently constructed an interesting chimera of human lysozyme and bovine $\alpha$-lactalbumin. In this chimera, amino acid residues 76-102 of 
lysozyme were replaced by $\alpha$-lactalbumin $72-97$, which represents the $\mathrm{Ca}^{2+}$-binding loop and the central helix $\mathrm{C}$. The chimera protein has acquired strong $\mathrm{Ca}^{2+}$-binding capacity and shows the ability to form a stable molten globule state under relatively mild conditions. Pardon et al. (79) have suggested that the peculiar aspartate-rich sequence of the $\mathrm{Ca}^{2+}$-binding site is one contribution to the stability of the molten globule state and that the $\mathrm{C}$ helix of the $\alpha$-lactalbumin molecule itself carries important features that determine the molten globule propensity.

\section{THE MOLTEN GLOBULE AS A MODEL TARGET OF MOLECULAR CHAPERONES}

Discovery of molecular chaperones has led to the understanding that various cell-biological phenomena are closely related to the problem of protein folding $(80,81)$. Among various chaperone proteins, the best-characterized chaperone from a physicochemical point of view is the Escherichia coli protein GroEL (14-mer of $60,000 \mathrm{Da}$ subunits), which is a member of the chaperonin 60 family. From their observation of in vitro folding of dihydrofolate reductase and rhodanese on the surface of GroEL, Hartl and co-workers (82-84) have suggested that the conformation of GroEL-bound polypeptides corresponds to the molten globule state and have proposed a model in which $E$. coli chaperones-DnaK, DnaJ, and GroEL-act successively along the pathway of chaperone-mediated protein folding in vivo. Because $\alpha$-lactalbumin exhibits the best-characterized molten globule state, this protein should provide a pertinent model to observed directly the interaction between GroEL and the molten globule state.

Okazaki et al. (85) have thus investigated the interactions between GroEL and two kinds of nonnative $\alpha$-lactalbumin-the molten globule state and the disulfide reduced form-by molecular sieve chromatography and hydrogen exchange measurements. The molten globule state was produced by removal of the bound $\mathrm{Ca}^{2+}$ at neutral $\mathrm{pH}$ where GroEL is in the fully native state. Disulfide-reduced $\alpha$-lactalbumin assumes a more relaxed and expanded structure than the molten globule state of the disulfide-intact protein (85). It is rather surprising that the results of Okazaki et al. (85) report that the interaction between GroEL and apo- $\alpha$-lactalbumin in the molten globule state cannot be observed by molecular sieve chromatography, which indicates that the interaction, if any, must be weak. Their results also show that disulfidereduced $\alpha$-lactalbumin is strongly bound to GroEL when $50 \mathrm{mM} \mathrm{KCl}$ is present. Therefore, the protein state recognized by GroEL may be more unfolded and expanded than the typical molten globule state of $\alpha$-lactalbumin.

Hayer-Hartl et al. (86) reported essentially the same experimental results of the interactions between GroEL and nonnative $\alpha$-lactalbumin as reported by Okazaki et al. (85), but their conclusion is apparently contradictory. Hayer-Hartl et al. (85) have concluded that GroEL inter- acts with the hydrophobic surfaces exposed by proteins in a flexible compact intermediate or molten globule state. The discrepancy between the conclusions is ascribable to different meanings of the term "molten globule" used by the two groups. Hayer-Hartl et al. (87) are using this term in a wider context, to include the conformational states of disulfide-reduced or disulfide-rearranged species of $\alpha$-lactalbumin. Such extension of the term is, however, very confusing and misleading (88). As we have seen before, an important characteristic of the molten globule state is its native-like tertiary fold, which is difficult to realize in the disulfide-reduced species and never realized in the disulfide-rearranged species.

Robinson et al. (89) have recently investigated the conformation of a three-disulfide derivative of $\alpha$-lactalbumin bound to GroEL by directly monitoring its hydrogen exchange kinetics using electrospray ionization mass spectrometry. The bound protein weakly protected amide protons from exchange to an extent closely similar to that of an uncomplexed molten globule state of the three-disulfide protein with native disulfide bonds, apparently suggesting that the protein bound to GroEL resembles a molten globule state. The three-disulfide derivative of $\alpha$ lactalbumin they used, however, again was the disulfiderearranged species. Moreover, the three-disulfide protein with native disulfides in the molten globule state is not strongly bound by GroEL (86), so that once the molecule acquires a native-like tertiary fold with native disulfide bonds, it may not be recognized strongly by GroEL. Recently, we have studied hydrogen exchange kinetics of tritium-labeled $\alpha$-lactalbumin with the four disulfide bonds being fully reduced, according to tritium-exchange measurements (Okazaki et al. unpublished data). The exchange kinetics measured in the absence and presence of GroEL have been found coincident with each other, although there is some secondary structure exhibiting protected amide protons in this partly folded state of $\alpha$-lactalbumin. Thus, in spite of strong binding of disulfide reduced $\alpha$-lactalbumin to GroEL, the secondary structure segments are not recognized and stabilized by GroEL. Therefore, what is recognized by GroEL is not the secondary structure nor a native-like tertiary fold, although both are typical characteristics of the molten globule state.

\section{CONCLUDING REMARKS}

This review summarizes recent progress in the studies of the molten globule state of $\alpha$-lactalbumin. The structural characteristics of the molten globule state of this protein are now much more clearly defined. The most important characteristic emerging from recent studies is the nativelike tertiary fold, the formation of which, at least in a part of the molecule, has also been suggested in the molten globule states of other globular proteins $(6,17,19$, 42-46) and is probably a common characteristic of the molten globule state for globular proteins similar in size 
$\alpha$-lactalbumin. It is also well documented that the number of tertiary folds that exist in natural globular proteins is not very large and most of the proteins are classified into only around 1000 protein families $(90,91)$. As it is likely that the number of tertiary folds is limited by physical constraints of protein structure (92), an important next step in protein folding studies is to elucidate the physical constraints or interactions that are necessary and sufficient to select specifically one of these tertiary folds for a particular protein. In this respect, studies of the molecular mechanism of stabilization of the molten globule state must be considered important.

It is also remarkable that the formation of the molten globule state from the fully unfolded state occurs very rapidly, within $10 \mathrm{~ms}$ after the refolding of $\alpha$-lactalbumin begins. Recently, we have reexamined the refolding reactions of $\alpha$-lactalbumin from the GdnHCl-induced, fully unfolded state by stopped-flow CD spectroscopy (Arai and K. Kuwajima, unpublished data). An early folding intermediate formed within the dead time $(\sim 10 \mathrm{~ms})$ of the stopped-flow measurements has the same spectral properties and the same stability against $\mathrm{GdnHCl}$-induced unfolding as the equilibrium molten globule state. Very rapid refolding within an interval of a few millisecond has recently been observed for several small globular proteins with approximately 60 amino acid residues (93-98), and it has been proposed that the direct folding to the native state is a much more rapid process than previously was expected $(98,99)$. Because the $\alpha$-helical domain of $\alpha$ lactalbumin is similar in size to these small globular proteins, the rapidity of formation of the molten globule state might be analogous to the rapidity of folding of these proteins. If this is the case, the slow folding of $\alpha$-lactalbumin may be rate-limited by rearrangement of the already partly organized $\alpha$-helical domain and the more unfolded $\beta$-sheet domain.

The experimental study by Griko et al. (66) and the theoretical calculation by Xie and Freire (74) seem to provide a reasonable interpretation of the thermodynamic characteristics of the molten globule state. Studies of the mutant proteins that stabilize or destabilize the molten globule state will provide a more concrete insight into the molecular interpretation of the thermodynamic properties and their relationship to the structure in the molten globule state. Kinetic refolding studies of these mutant proteins must be important for answering the question whether the molten globule is an obligatory intermediate in kinetic folding or is merely a species produced by a kinetic trap. Despite great advances in our understanding of the molten globule state, this important question remains unsolved.

The molten globule state and the disulfide-reduced form of $\alpha$-lactalbumin have been shown to be good model targets for the chaperonin GroEL. The interaction between GroEL and the molten globule of disulfide-intact $\alpha$-lactalbumin has recently been investigated more quantitatively by comparing the refolding kinetics of the protein in the absence and presence of GroEL (100; K.
Katsumata et al., unpublished data). The binding constant estimated as around $10^{5} \mathrm{M}^{-1}\left(\mathrm{pH} 7\right.$ and $25^{\circ} \mathrm{C}$ at 50 $\mathrm{mM}$ sodium cacodylate) is shown to be two orders of magnitude smaller than the binding constant between GroEL and disulfide-reduced $\alpha$-lactalbumin and too weak to detect by molecular sieve chromatography. Although such chaperone studies are not directly connected with elucidation of the molecular mechanism of stabilization of the molten globule state, the studies are important for understanding the relationships between the in vitro and in vivo folding reactions. Many other chaperone proteins are required in the correct folding of a protein in vivo $(80$, 81 ), and physicochemical studies of the interactions between the chaperone proteins and a model target will become progressively important. The well-characterized molten globule state of $\alpha$-lactalbumin will also be useful in such studies, and will be the favorite among many protein folders.

Note added in proof: After completion of this article, a few interesting papers (101-103) concerning the molten globule state of $\alpha$-lactalbumin have been published. The results described in these papers are consistent with the picture of the molten globule described here.

This work was supported by Grants-in-Aid for Scientific Research from the Ministry of Education, Science and Culture of Japan, and by a grant (RG-331/93M) from the International Human Frontier Science Program (HFSP) Organization.

\section{REFERENCES}

1. Levinthal, C. (1968) Are there pathways for protein folding? J. Chim. Phys. 65, 44-45

2. Kim, P. S., and Baldwin, R. L. (1982) Specific intermediates in the folding reactions of small proteins and the mechanism of protein folding. Annu. Rev. Biochem. 51, 459-489

3. Kim, P. S., and Baldwin, R. L. (1990) Intermediates in the folding reactions of small proteins. Annu. Rev. Biochem. 59, 631-660

4. Kuwajima, K. (1989) The molten globule state as a clue for understanding the folding and cooperativity of globular-protein structure. Proteins 6, 87-103

5. Dobson, C. M. (1991) Characterization of protein folding intermediates. Curr. Opin. Struct. Biol. 1, 22-27

6. Baldwin, R. L. (1993) Pulsed H/D-exchange studies of folding intermediates. Curr. Opin. Struct. Biol. 3, 84-91

7. Matthews, C. R. (1993) Pathways of protein folding. Annu. Rev. Biochem. 62, 653-683

8. Baldwin, R. L. (1994) Finding intermediates in protein folding. BioEssays 16, 207-210

9. Ptitsyn, 0. B. (1994) Kinetic and equilibrium intermediates in protein folding. Protein Eng. 7, 593-596

10. Fink, A. L. (1995) Compact intermediate states in protein folding. Annu. Rev. Biophys. Biomol. Struct. 24, 495-522

11. Barrick, D., and Baldwin, R. L. (1993) The molten globule intermediate of apomyoglobin and the process of protein folding. Protein Sci. 2, 869-876

12. Kuwajima, K., Nitta, K., Yoneyama, M., and Sugai, S. (1976) Three-state denaturation of $\alpha$-lactalbumin by guanidine hydrochloride. J. Mol. Biol. 106, 359_373

13. Privalov, P. L. (1979) Stability of proteins. Small globular proteins. Adv. Protein Chem. 33, 167-241

14. Creighton, T. E. (1979) Electrophoretic analysis of the unfolding of proteins by urea. J. Mol. Biol. 129, 235-264

15. Kuwajima, K. (1995) Circular dichroism. In Protein Stability and Folding: Methods in Molecular Biology, Vol. 40 (Shirley, B. A., ed) pp. 115-135. Humana Press Inc., Totowa, N.J.

16. Kuwajima, K. (1995) Stopped-flow circular dichroism. In Circular Dichroism and the Conformational Analysis of Biomolecules (Fasman, G. D., ed) Plenum, New York In press

17. Englander, S., W., and Mayne, L. (1992) Protein folding studied using hydrogen-exchange labeling and two-dimensional NMR. Annu. Rev. Biophys. Biomol. Struct. 21, 243-265 
18. Dobson, C. M. (1992) Unfolded proteins, compact states and molten globules. Curr. Opin. Siruct. Biol. 2, 6-12

19. Roder, H., and Elöve, G. A. (1994) Early stages of protein folding. In Mechanisms of Protein Folding (Pain, R. H., ed) pp. 26-54, IRL Press, Oxford

20. Dobeon, C. M. (1994) Protein folding: Solid evidence for molten globules. Curr. Biol. 4, 636-640

21. Baldwin, R. L. (1995) The nature of protein folding pathways: The classical versus the new view. J. Biomol. NMR 5, 103-109

22. Hill, R. L., and Brew, K. (1975) Lactose synthetase. Adv. Enzymol. Relat. Areas Mol. Biol. 43, 411-490

23. Hiraoka, Y., Segawa, T., Kuwajima, K., Sugai, S., and Murai, N. (1980) $\alpha$-Lactalbumin: A calcium metalloprotein. Biochem. Biophys. Res. Commun. 95, 1098-1104

24. McKenzie, H. A., and White, F. H., Jr. (1991) Lysozyme and $\alpha$-lactalbumin: Structure, function, and interrelationships. Adv. Provein Chem. 41, 173-315

25. Qasba, P. K., and Safaya, S. K. (1984) Similarity of the nucleotide sequences of rat $\alpha$-lactalbumin and chicken lysozyme genes. Nature (London) 308, $377-380$

26. Nitta, K., and Sugai, S. (1989) The evolution of lysozyme and $\alpha$-lactalbumin. Eur. J. Biochem. 182, 111-118

27. Acharya, K. R., Stuart, D. I., Walker, N. P. C., Lewis, M., and Phillips, D. C. (1989) Refined structure of baboon $\alpha$-lactalbumin at $1.7 \AA$ resolution. Comparison with c-type lysozyme. J. Mol. Biol. 208, 99-127

28. Acharya, K. R., Ren, J., Stuart, D. I., Phillips, D. C , and Fenna, R. E. (1991) Crystal structure of human $\alpha$-lactalbumin at 1.7 resolution. J. Mol. Biol. 221, 571-581

29. Harata, K., and Muraki, M. (1992) X-ray structural evidence for a local helix-loop transition in $\alpha$-lactalbumin. J. Biol. Chem. 267, 1419-1421

30. Ren, J., Stuart, D. I., and Acharya, K. R. (1993) $\alpha$-lactalbumin possesses a distinct zinc binding site. J. Biol. Chem. 268, 19292-19298

31. Kronman, M. J. (1989) Metal-ion binding and the molecular conformational properties of $\alpha$-lactalbumin. CRC Crit. Rev. Biochem. Mol. Biol. 24, $565-667$

32. Nitta, K., Kita, N., Kuwajima, K., and Sugai, S. (1977) Equilibrium and kinetics of the unfolding of $\alpha$-lactalbumin by guanidine hydrochloride (IV). Dependence of the $\mathrm{N} \rightleftharpoons \mathrm{A}$ transconformation on temperature. Biochim. Biophys. Acta 490, 200-208

33. Nozaka, M., Kuwajima, K., Nitta, K., and Sugai, S. (1978) Detection and characterization of the intermediate on the folding pathway of human $\alpha$-lactalbumin. Biochemistry 17, 3753-3758

34. Kuwajima, K. (1977) A folding model of $\alpha$-lactalbumin deduced from the three-state denaturation mechanism. J. Mol. Biol. 114, 241-258

35. Kuwajima, K., Hiraoka, Y., Ikeguchi, M., and Sugai, S. (1985) Comparison of the transient folding intermediates in lysozyme and $\alpha$-lactalbumin. Biochemistry 24, 874-881

36. Ikeguchi, M., Kuwajima, K., Mitani, M., and Sugai, S. (1986) Evidence for identity between the equilibrium unfolding intermediate and a transient folding intermediate: $A$ comparative study of the folding reactions of $\alpha$-lactalbumin and lysozyme. Biochemistry 25, 6965-6972

37. Kuwajima, K. (1992) Protein folding in vitro. Curr. Opin. Biotechnol. 3, $462-467$

38. Sugai, S., and Ikequchi, M. (1994) Conformational comparison between $\alpha$-lactalbumin and lysozyme. Adv. Biophys. 30, 37-84

39. Baum, J., Dobson, C. M., Evans, P. A., and Hanley, C. (1989) Characterization of a partly folded protein by NMR methods: Studies on the molten globule state of guinea pig $\alpha$-lactalbumin. Biochemistry 28, 7-13

40. Alexandrescu, A. T., Evans, P. A., Pitkeathly, M., Baum, J., and Dobson, C. M. (1993) Structure and dynamics of the acid-denatured molten globule state of $\alpha$-lactalbumin: A two-dimensional NMR study. Biochemistry 32, 1707-1718

41. Chyan, C. L., Wormald, C., Dobson, C. M., Evans, P. A., and Baum, J. (1993) Structure and stability of the molten globule state of guinea-pig $\alpha$-lactalbumin: A hydrogen exchange study. Biochemistry 32, 5681-5691

42. Jennings, P. A., and Wright, P. E. (1993) Formation of a molten globule intermediate early in the kinetic folding pathway of apomyoglobin. Science 262, 892-896

43. Jeng, M. F., Englander, S. W., Elöve, G. A., Wand, A. J., and Roder, H. (1990) Structural description of acid-denatured cytochrome $c$ by hydrogen exchange and 2D NMR. Biochemistry 29, 10433-10437

44. Mullins, L. S., Pace, C. N., and Raushel, F. M. (1993) Investigation of ribonuclease $\mathrm{Tl}$ folding intermediates by hydrogen-deuterium amide exchange-two-dimensional NMR spectroscopy. Biochemistry 32, 6152-6156

45. Jacobs, M. D., and Fox, R. O. (1994) Staphylococcal nuclease folding intermediate characterized by hydrogen exchange and NMR spectroscopy. Proc. Natl. Acad. Sci. USA 91, 449-453

46. Jones, B. E., and Matthews, C. R. (1995) Early intermediates in the folding of dihydrofolate reductase from Escherichia coli detected by hydrogen exchange and NMR. Protein Sci. 4, 167-177

47. Shiraki, K., Nishikawa, K., and Goto, Y. (1995) Trifluoroethanol-induced stabilization of the $\alpha$-helical structure of $\beta$-lactoglobulin: Implication for non-hierarchical protein folding. J. Mol. Biol. 245, 180-194

48. Improta, S., Molinari, H., Pastore, A., Consonni, R., and Zetta, L. (1995) Probing protein structure by solvent perturbation of NMR spectra-Photochemically induced dynamic nuclear polarization and paramagnetic perturbation techniques applied to the study of the molten globule state of $\alpha$-lactalbumin. Eur. J. Biochem. 227, 87-96
49. Hamada, S., Moriyama, Y., Yamaguchi, K., and Takeda, K. (1994) Conformational stability of $\alpha$-lactalbumin missing a peptide bond between Asp66 and Pro67. J. Protein Chem. 13, 423-428

50. Nölting, B., Jiang, M., and Sligar, S. G. (1993) The acidic molten globule state of $\alpha$-lactalbumin probed by sound velocity. J. Am. Chem. Soc. 115 , 9879-9882

51. Ikeguchi, M., Sugai, S., Fujino, M., Sugawara, T., and Kuwajima, K. (1992) Contribution of the 6-120 disulfide bond of $\alpha$-lactalbumin to the stabilities of its native and molten globule states. Biochemistry 31, 12695-12700

52. Ewbank, J. J., and Creighton, T. E. (1991) The molten globule protein conformation probed by disulphide bonds. Nature (London) 350, 518-520

53. Ewbank, J. J., and Creighton, T. E. (1993) Structural characterization of the disulfide folding intermediates of bovine $\alpha$-lactalbumin. Biochemistry 32 , 3694-3707

54. Creighton, T. E., and Ewbank, J. J. (1994) Disulfide-rearranged molten globule state of $\alpha$-lactalbumin. Biochemistry 33, 1534-1538

55. Peng, Z., and Kim, P.S. (1994) A protein dissection study of a molten globule. Biochemistry 33, 2136-2141

56. Wu, L. C., Peng, Z., and Kim, P. S. (1995) Bipartite structure of the $\alpha$-lactalbumin molten globule. Nature Struct. Biol. 2, 281-286

57. Peng, Z. Y., Wu, L. C., Schulman, B. A., and Kim, P. S. (1995) Does the molten globule have a native-like tertiary fold? Philos. Trans. $R$. Soc. Lond. Biol. Sci. 348, 43-47

58. Peng, Z., Wu, L. C., and Kim, P. S. (1995) Local structural preferences in the $\alpha$-lactalbumin molten globule. Biochemistry 34, 3248-3252

59. Pfeil, W., Bychkova, V. E., and Ptitsyn, O. B. (1986) Physical nature of the phase transition in globular proteins. Calorimetric study of human $\alpha$-lactalbumin. FEBS Lett. 198, 287-291

60. Dolgikh, D. A., Abaturov, L. V., Bolotina, I. A., Brazhnikov, E. V., Bychkova, V. E., Bushuev, V. N., Gilmanshin, R. I., Levedev, Y. O., Semisotnov, G. V., Tiktopulo, E. I., and Ptitsyn, O. B. (1985) Compact state of a protein molecule with pronounced small-scale mobility: bovine $\alpha$-lactalbumin. Eur. Biophys. J. 13, 109-121

61. Dolgikh, D. A., Gilmanshin, R. I., Brazhnikov, E. V., Bychkova, V. E. Semisotnov, G. V., Venyaminov, S. Y., and Ptitsyn, O. B. (1981) $\alpha$-Lactalbumin: Compact state with fluctuating tertiary structure? FEBS Lett. 136 , 311-315

62. Xie, D., Bhakuni, V., and Freire, E. (1991) Calorimetric determination of the energetics of the molten globule intermediate in protein folding: Apo- $\alpha$-lactalbumin. Biochemistry 30, 10673-10678

63. Yutani, K., Ogasahara, K., and Kuwajima, K. (1992) The absence of the thermal transition in apo- $\alpha$-lactalbumin in the molten globule state: $A$ study by differential scanning microcalorimetry. J. Mol. Biol. 228, 347-350

64. Xie, D., Bhakuni, V., and Freire, E. (1993) Are the molten globule and the unfolded states of apo- $\alpha$-lactalbumin enthalpically equivalent? J. Mol. Biol. 232, 5-8

65. Hiraoka, Y., and Sugai, S. (1985) Equilibrium and kinetic study of sodiumand potassium-induced conformational changes of apo- $\alpha$-lactalbumin. Int. J. Pept. Procein Res. 26, 252-261

66. Griko, Y. V., Freire, E., and Privalov, P. L. (1994) Energetics of the $\alpha$-lactalbumin states: $A$ calorimetric and statistical thermodynamic study. Biochemistry 33, 1889-1899

67. Ptitsyn, O. B., and Uversky, V. N. (1994) The molten globule state is a third thermodynamical state of protein molecules. FEBS Lett. 341, 15-18

68. Lala, A. K., and Kaul, P. (1992) Increased exposure of hydrophobic surface in molten globule state of $\alpha$-lactalbumin. Fluorescence and hydrophobic photolabeling studies. J. Biol. Chem. 267, 19914-19918

69. Vanderheeren, G., and Hanssens, I. (1994) Thermal unfolding of bovine $\alpha$-lactalbumin. Comparison of circular dichroism with hydrophobicity measurements. J. Biol. Chem. 269, 7090-7094

70. Eynard, L., Iametti, S., Relkin, P., and Bonomi, F. (1992) Surface hydrophobicity changes and heat-induced modifications of $\alpha$-lactalbumin. J. Agr. Food Chem. 40, 1731-1736

71. Morozova, L., Desmet, J., and Joniau, M. (1993) Complexes of the polyamines spermine, spermidine and putrescine with $\alpha$-lactalbumins. Eur. J. Biochem. 218, 303-309

72. Apenten, R. K. 0. (1995) A three-state heat-denaturation of bovine $\alpha$-lactalbumin. Food Chem. 52, 131-133

73. Shimizu, A., Ikeguchi, M., and Sugai, S. (1993) Unfolding of the molten globule state of $\alpha$-lactalbumin studied by 'H-NMR. Biochemistry 32. 13198-13203

74. Xie, D., and Freire, E. (1994) Molecular basis of cooperativity in protein folding. V. Thermodynamic and structural conditions for the stabilization of compact denatured states. Proteins 19, 291-301

75. Xie, D., and Freire, E. (1994) Structure based prediction of protein folding intermediates. J. Mol. Biol. 242, 62-80

76. Freire, E., and Xie, D. (1994) Thermodynamic prediction of structural determinants of the molten globule state of barmase. Biophys. Chem. 51 , 243-251

77. Xie, D., Fox, R., and Freire, E. (1994) Thermodynamic characterization of an equilibrium folding intermediate of staphylococcal nuclease. Protein Sci. 3, 2175-2184

78. Uchiyama, H., Perez-Prat, E. M., Watanabe, K., Kumagai, I., and Kuwajima, K. (1995) Effects of amino acid substitutions in the hydrophobic core of $\alpha$-lactalbumin on the stability of the molten globule state. Protein Eng. In press 
79. Pandon, E. Haezebrouck, P. De Baetselier, A., Hooke, S. D., Fancourt, K. T., Desmet, J., Dobson, C. M., Van Dael, H., and Joniau, M. (1995) A $\mathrm{Ca}^{2+}$-binding chimera of human lysozyme and bovine $\alpha$-lactalbumin that can form a molten globule. J. Biol. Chem. 270, 10514-10524

80. Ellis, R. J. (1993) The general concept of molecular chaperones. Philos. Trans. R. Soc. Lond. Biol. Sci. 339, 257-261

81. Hendrick, J. P., and Hartl, F. U. (1993) Molecular chaperone functions of heat-shock proteins. Annu. Rev. Biochem. 62, 349-384

82. Martin, J., Langer, T., Boteva, R., Schramel, A., Horwich, A. L., and Hartl, F. U. (1991) Chaperonin-mediated protein folding at the surface of groEL through a "molten globule"-like intermediate. Nature (London) 352, 36-42

83. Langer, T., Lu, C., Echols, H., Flanagan, J., Hayer, M. K., and Hartl, F. U. (1992) Successive action of DnaK, DnaJ and GroEL along the pathway of chaperone-mediated protein folding. Nature (London) 356, 683-689

84. Hartl, F. U., Martin, J., and Neupert, W. (1992) Protein folding in the cell: The role of molecular chaperones Hsp70 and Hsp60. Annu. Rev. Biophys. Biomol. Struct. 21, 293-322

85. Okazaki, A., Ikura, T., Nikaido, K., and Kuwajima, K. (1994) The chaperonin GroEL does not recognize apo- $\alpha$-lactalbumin in the molten globule state. Nature Struct. Biol. 1, 439-446

86. Hayer-Hartl, M. K., Ewbank, J. J., Creighton, T. E., and Hartl, F. U. (1994) Conformational specificity of the chaperonin GroEL for the compact folding intermediates of $\alpha$-lactalbumin. EMBO J. 13, 3192-3202

87. Ewbank, J. J., Creighton, T. E., Hayer-Hartl, M. K., and Hartl, F. U. (1995) What is the molten globule? Nature Struct. Biol. 2, 10

88. Okazaki, A., Ikura, T., and Kuwajima, K. (1995) What is the molten globule? Reply. Nature Struct. Biol. 2, 10-11

89. Rohinson, C.V.,Gross, M., Eyles, S. J Ewhank, J.J Mayhew, M., Hartl, F. U., Dobson, C. M., and Radford, S. E. (1994) Conformation of GroEL-bound $\alpha$-lactalbumin probed by mass spectrometry. Nature (London) 372,646-651

90. Chothia, C. (1992) One thousand families for the molecular biologist. Nature (London) 357, 543-544

91. Orengo, C. A., Jones, D. T., and Thornton, J. M. (1994) Protein superfamilies and domain superfolds. Nature (London) 372, 631-634

92. Ptitsyn, O. B., and Finkelstein, A. V. (1980) Similarities of protein topologies: evolutionary divergence, functional convergence or principles of folding? $Q$. Rev. Biophys. 13, 339-386
93. Jackson, S. E., and Fersht, A. R. (1991) Folding of chymotrypsin inhibitor 2. 2. Influence of proline isomerization on the folding kinetics and thermodynamic characterization of the transition state of folding. Biochemistry 30 , 10436-10443

94. Alexander, P., Orban, J., and Bryan, P. (1992) Kinetic analysis of folding and unfolding the 56 amino acid IgG-binding domain of streptococcal protein G. Biochemistry 31, 7243-7248

95. Kuszewski, J., Clore, G. M., and Gronenborn, A. M. (1994) Fast folding of a prototypic polypeptide: The immunoglobulin binding domain of streptococcal protein G. Protein Sci. 3, 1945-1952

96. Otzen, D. E. Itzhaki, L. S., ElMasry, N. F. Jackson, S. E and Fersht, A. R. (1994) Structure of the transition state for the folding/unfolding of the barley chymotrypsin inhibitor 2 and its implications for mechanisms of protein folding. Proc. Natl. Acad. Sci. USA 91, 10422-10425

97. Huang, G. S., and Oas, T. G. (1995) Submillisecond folding of monomeric lambda repressor. Proc. Natl. Acad. Sci. USA 92, 6878-6882

98. Schindler, T., Herrier, M., Marahiel, M. A., and Schmid, F. X. (1995) Extremely rapid protein folding in the absence of intermediates. Nature Struct. Biol. 2, 663-673

99. Sosnick, T. R., Mayne, L., Hiller, R., and Englander, S. W. (1994) The barriers in protein folding. Nature Struct. Biol. 1, 149-156

100. Katsumata, K., Okazaki, A., and Kuwajima, K. (1995) Effect of the chaperonin GroEL on the refolding kinetics of $\alpha$-lactalbumin. The 10 h Rinshoken International Conference: Stress Response and Molecular Chaperones, May 8-11, 1995, Chiba, Japan; Program Abstracts, p. 59

101. Balach, J., Forege, V., van Nuland, N.A.J., Winder, S.L., Hore, P.J., and Dobson, C.M. (1995) Following protein folding in real time using NMR spectroscopy. Nature Struct. Biol. 2, 865-870

102. Polverino de Laureto, P., De Filippis, V., Di Bello, M., Zambonin, M., and Fontana, A. (1995) Probing the molten globule state of $\alpha$-lactalbumin by limited proteolysis. Biochemistry 34, 12596-12604

103. Schulman, B.A., Redfield, C., Peng, Z., Dobson, C.M. and Kim, P.S. (1995) Different subdomains are most protected from hydrogen exchange in the molten globule and native states of $\alpha$-lactalbumin. J. Mol. Bio. 253, $651-657$

\title{
A Series of Reviews now in The FASEB Journal
}

\author{
Flavoprotein Structure and Mechanism
}

\section{Coordinator: Vincent Massey}

T. O. Baldwin. Bacterial luciferase

structure and function

G. T. Gassner, M. L. Ludwig, and D. P. Ballou. Phthalate dioxygenase and phthalate dioxygenase reductase

B. Entsch and W. J. H. van Berkel.

Structure and mechanism of p-hydroxybenzoate hydroxylase

R. Hille and T. Nishino. Xanthine oxidase and xanthine dehydrogenase interconvertible forms of the same enzyme

$P$. A. Karplus and V. Massey. Old yellow enzyme: structure and function

\section{R.L. Krauth-Siegel and R. Schonek.}

Trypanathione reductase and lipoamide dehydrogenase as targets for a structurebased drug design

K. A. White and M. A. Marletta. Nitric oxide synthase

E. Pai. Overview of flavoprotein crystal structures

T. P. Singer and R. Ramsay. Monoamine oxidase: old friends hold many surprises C. Thorpe and J.-J. P. Kim. Structure and mechanism of action of the acyl-CoA dehydrogenases

C. H. Williams, Jr. Mechanism and structure of thioredoxin reductase thioredoxin reductase 